Artículo de revisión

\title{
Fracturas de cuello y diáfisis femoral ipsilateral: revisión de la literatura.
}

\author{
Ortega-Yago, A ${ }^{1 *}$, Balfagón-Ferrer, A ${ }^{2}$, Alonso-Caravaca, A $^{3}$, Castillo-Soriano, MA ${ }^{4,}$ De Pedro-Abascal, ${ }^{5}$ \\ 1 * Correspondence: ortegayago94@gmail.com \\ 1,2,3 Department of Orthopedic Surgery and Traumatology, The University and Polytechnic la Fe Hospital of \\ Valencia.
}

Academic Editor: Ortega-Yago, A. Received: 10 January 2021.

Accepted:

Published:

Copyright: (c) 2021 by the authors. Submitted for possible open access publication under the terms and conditions of the Creative Commons Attribution (CC BY) license (http://creativecommons.org/licenses /by/4.0/).

\begin{abstract}
: las fracturas de cuello y diáfisis femoral ipsilateral son fracturas poco frecuentes, que se asocian a pacientes jóvenes tras un mecanismo de alta energía. Este tipo de fracturas suponen un reto a la hora de su diagnóstico, dado que hasta un $20 \%$ de las fracturas de cuello femoral pueden pasar desapercibidas. La correcta planificación quirúrgica tras un diagnóstico preciso permite un tratamiento óptimo de las fracturas, al igual que una disminución de la tasa de complicaciones derivadas de las mismas. Actualmente con el avance de los implantes, existen múltiples opciones quirúrgicas para tratar estas fracturas, y que permite individualizar el tratamiento dependiendo del paciente y del tipo de fractura que tiene. Esta revisión tiene como objetivo sintetizar los conceptos acerca del diagnóstico y manejo quirúrgico de las fracturas ipsilaterales de cuello y diáfisis femorales.
\end{abstract}

Keywords: "fracturas diafisarias de femur", "fracturas de cuello femoral", "diagnóstico precoz"

\section{Introduction}

Las fracturas de cuello y diáfisis femoral ipsilateral son fracturas infrecuentes (2-6\%). La edad media es de 35 años aproximadamente, y más de la mitad de los casos se producen en hombres. Estas fracturas se producen por mecanismo de alta energía, en el cual existe un impacto de alta energía sobre la rodilla. Posteriormente, la energía entra por la rodilla creando una fuerza axial por la diáfisis hasta llegar al cuello femoral. [1,2]. Muchas veces se produce porque el miembro inferior está en abducción, a diferencia de cuando está en adducción, que tiende a producirse luxación posterior de cadera [3].

Las fracturas de cuello femoral que se asocian más frecuentemente a las fracturas ipsilaterales de diáfisis son las subcapitales (Figura 1) o las basicervicales no desplazadas, y las fracturas de cuello vertical (Pauwels tipo 3).

En muchas ocasiones existe conminación en la diáfisis femoral. Además, estas fracturas están asociadas a lesiones de rodilla ipsilaterales (20-40\%), entre las que se encuentran fracturas de meseta tibial, lesiones de complejos ligamentosos, fractura de rótula o luxación de rodilla. $[4,5]$ 

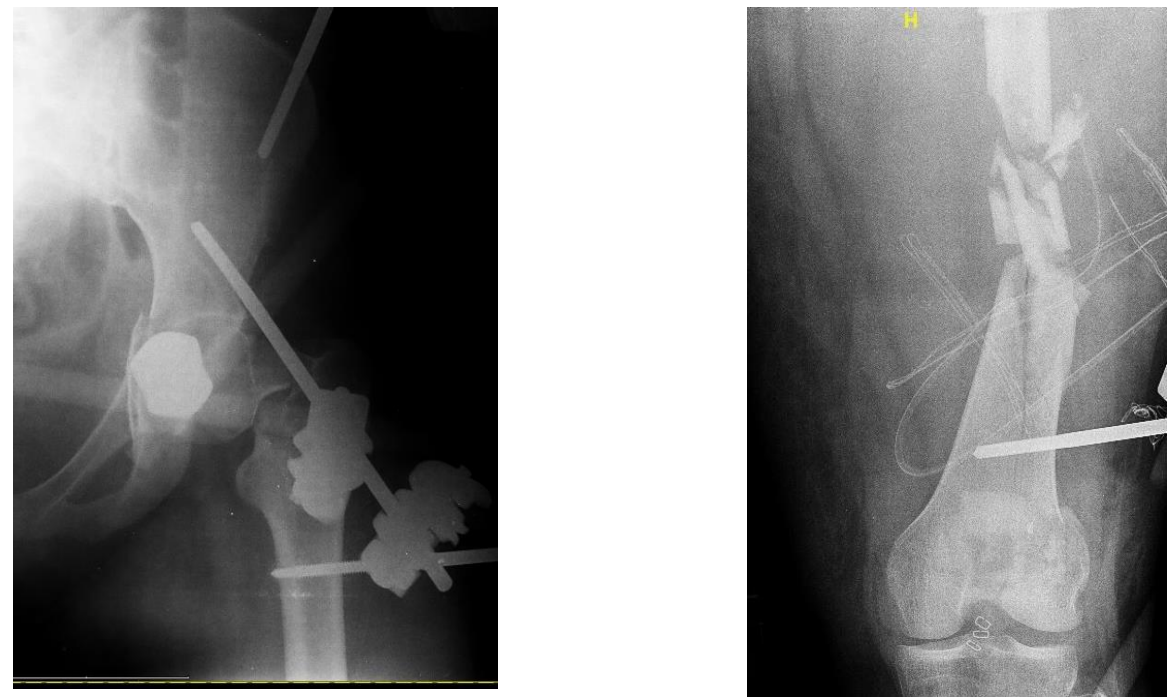

Figura 1: fractura subcapital y diafisaria de fémur izquierdo

\section{Diagnóstico}

El diagnóstico suele ser tardío y supone un reto para el traumatólogo, dado que puede pasar desapercibido en muchas ocasiones. Entre estos motivos, está el fémur, que tiende a la rotación externa. Otros factores añadidos son la falta de radiografías, la mala calidad de las mismas, el poco desplazamiento femoral del cuello, y la difícil exploración en pacientes inestables del paciente. Hasta el $20 \%$ de estas fracturas pueden pasar totalmente desapercibidas [3].

Para evitar que esto suceda, hemos de obtener una radiografía anteroposterior de pelvis, así como axial de cadera y lateral de diáfisis femoral. Con respecto, al uso del TC, prueba cada vez más utilizada en nuestro medio para planificación quirúrgica y diagnóstico de fracturas, se ha observado que es de gran utilidad para refinar el diagnóstico y descubrir fracturas ocultas.

Yang et al observaron que el $76 \%$ de las fracturas que no eran detectadas por las radiografías, sí fueron diagnosticadas gracias al TC. [6]. Conforme el uso del TC se fue extendiendo y los cortes fueron disminuyendo de grosor de milímetros, el diagnóstico ha conseguido precisarse más, llegando hasta el 82\% de la precisión diagnóstica [7].

Existen actualmente algunos protocolos de diagnóstico de imagen, entre los que se encuentran radiografías en rotación interna, la realización de un TC, controles fluoroscópicos prequirúrgicamente, previo a la fijación de diáfisis y tras la fijación de la misma, así como la realización de radiografía postintervención. Con ese protocolo, se reduce un $90 \%$ la probabilidad de que pase desapercibida una fractura oculta 


\section{3. ¿Cuándo intervenir al paciente?}

Existen recomendaciones para decidir cuándo es el momento idóneo para intervenir al paciente. Normalmente se aconseja que sea de forma urgente, nada más el paciente esté estable, dado que permite recuperar la vascularización femoral lo antes posible. Aun así, no existe un consenso sobre cuándo realizar la cirugía, dado que estas fracturas son raras, los patrones de fracturas son diferentes dependiendo de los pacientes, hay gran variabilidad de tratamientos, y las cohortes son pequeñas [3]

\section{Tratameinto quirúrgico}

En el tratamiento quirúrgico hay dos objetivos principales de realizar la cirugía: la reducción anatómica de la cabeza femoral, y la reducción de la diáfisis para corregir longitud, alineamiento y rotación.

Muchos autores coinciden en que la cabeza femoral tiene preferencia sobre el tratamiento de la diáfisis. Primero, porque las complicaciones de la cabeza femoral son mayores que la diáfisis (necrosis avascular, ausencia de consolidación), y segundo, porque si se reduce la diáfisis femoral primero, la reducción de la cabeza femoral es más complicada que si se realiza a la inversa [4,5]. Si no se pueda realizar una reducción cerrada de la cabeza femoral, está indicada la reducción abierta para realizar una reducción lo más anatómica posible.

En cuanto a las opciones de la osteosíntesis disponible para estas fracturas, tenemos el implante único y la combinación de dos implantes.

Con respecto a un implante, la reducción tiende a ser más compleja. Es preferible el uso de implante único si el cuello está mínimamente desplazado (Figura 2).

En cuanto a la combinación de dos implantes, la reducción del cuello tiende a ser algo más sencilla, y se prefiere cuando el cuello femoral está desplazado.

Entre las opciones de implante único tenemos el clavo cefalomedular (clavo de reconstrucción) y el DHS largo. La LCP no está preparada para fijar el cuello femoral por la incapacidad que tiene de realizar compresión. En algunos estudios se ha observado que el enclavado medular tiene mayor tasa de ausencia de consolidación con respecto a la combinación de dos implantes [9]. 


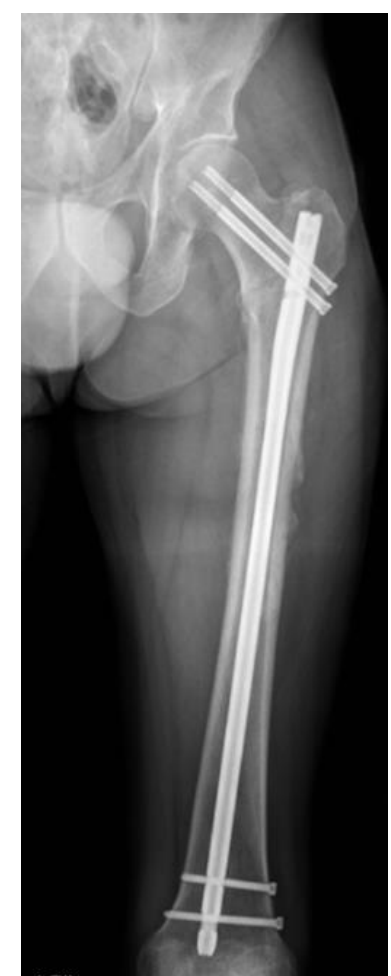

Figura 2: Clavo de reconstrucción, un implante.

Con respecto al uso de dos implantes, existen tres opciones:

- Clavo anterógrado + canulados: existe menos espacio para trabajar la reducción de la cabeza (Figura 3 y 4 ).

- Clavo retrógrado + DHS/ canulados: más trabajo para la reducción del cuello femoral (Figura 5)

- $\quad$ Placa de compresión / MIPO + DHS/ canulados 


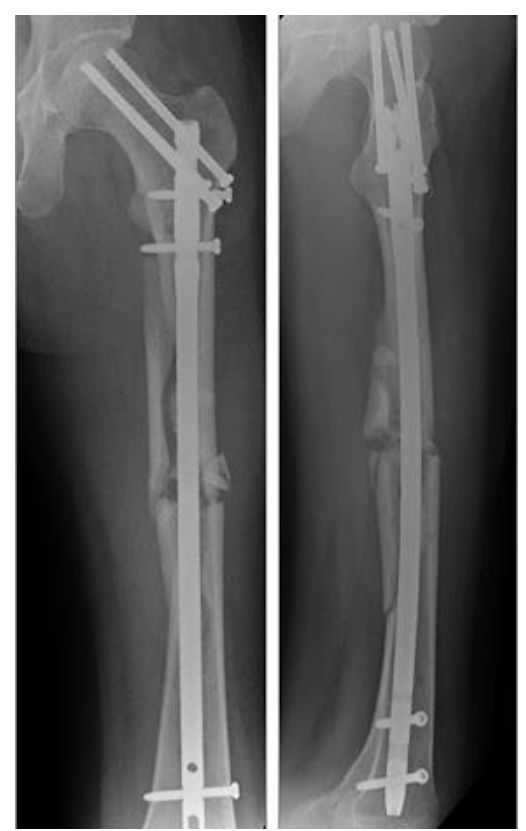

Figura 3 y 4: clavo anterógrado junto con tornillos canulados.

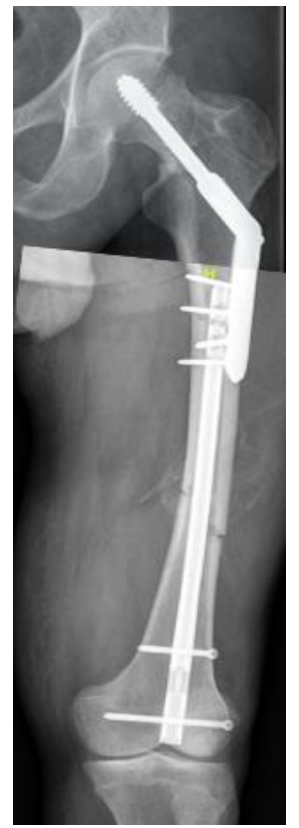

Figura 5: clavo retrógrado junto con DHS

Para el cuello femoral combinado con clavo anterógrado o retrógrado, se pueden utilizar diferentes opciones: $[9,10,11]$

- Femoral Nail system: es una opción cuando existen fracturas subcapitales. Contraindicado su uso en intertrocantéricas o pertrocantéricas.

- $\quad$ Tornillos canulados: para fracturas subcapitales

- DHS: para fracturas pertrocantéreas, intertrocantéreas o basicervicales. 
- DHS + canulados: En el caso de las Pauwels tipo 3, se puede utilizar combinados tanto el DHS como los canulados por el patrón vertical que presentan, y porque el DHS de forma aislada tiene dificultades para aguantar las fuerzas rotacionales en este patrón de fracturas. [12]
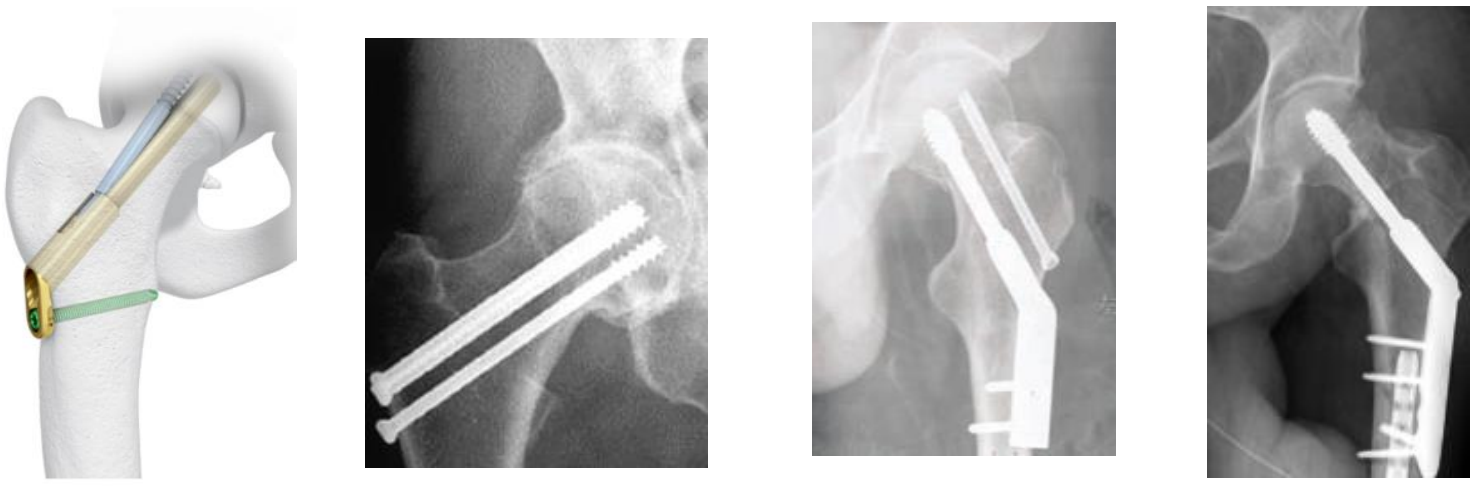

Figura 4: distintas opciones para fijar el cuello femoral: femoral nail system (A), tornillos canulados (B), DHS (C) y DHS junto con canulado (D).

No existen diferencias significativas entre el DHS y los tornillos canulados en cuanto a aumento de probabilidad de ausencia de consolidación, ni en la funcionalidad posterior del paciente. [10]

Las actuales recomendaciones aconsejan que se frese previo a introducir el clavo, dado que se ha visto que fresar disminuye la probabilidad de ausencia de consolidación, especialmente en la diáfisis. [5,10]. Esto se produce porque al no haber fresado, el clavo no se adapta dado que el componente del eje del clavo suele ser de tamaño inferior en comparación con los diámetros del canal medular.

El tratamiento definitivo de estas fracturas, dependerá del si el diagnóstico se hace antes, mientras o después de la fijación, o si la fractura de cuello femoral está desplazada.

Si la cabeza está mínimamente desplazada o sin desplazar, se tiende a intentar utilizar un implante. Para ello, se fija primero la cabeza de forma temporal, se coloca posteriormente el clavo en la diáfisis, y finalmente se fija de forma definitiva la cabeza.

Si la cabeza está desplazada, se tiende a utilizar dos implantes. Primero se fija la cabeza femoral con reducción cerrada o bien abierta, y posteriormente se reduce la diáfisis. De esa forma consigues manipular la diáfisis con el cuello femoral fijo.

Entre las técnicas que pueden utilizarse para fijar el cuello femoral está la "miss a nail". Esta técnica permite reducir la fractura de cuello femoral antes de fijar la diáfisis femoral. Permite estabilizar de forma temporal, bien con agujas (de forma anterior) o bien reducción abierta si es preciso el cuello femoral bajo control de escopia. Posteriormente se introduce 
un clavo de manipulación, se coloca un adaptador (miss a nail) y luego se introduce una guía. Luego se coloca una aguja para mantener la posición y estabilizar la fractura.

Se coloca bajo escopia las agujas guía para posteriormente insertar los tornillos perpendiculares a la fractura. Posteriormente se retira el clavo de manipulación y se determina la longitud de los tornillos, para luego introducirlos.

Si el hallazgo de la fractura es intraoperatorio tras la inserción del clavo endomedular, se pueden añadir tornillos canulados anteriores al clavo ( 2 implantes), o bien cambiar el clavo que se está utilizando a un clavo de reconstrucción. En el caso de que el hallazgo sea postoperatorio, dependiendo del tipo de fractura y del implante previo habremos de utilizar otro implante o bien modificar el implante previo (Figura 5).
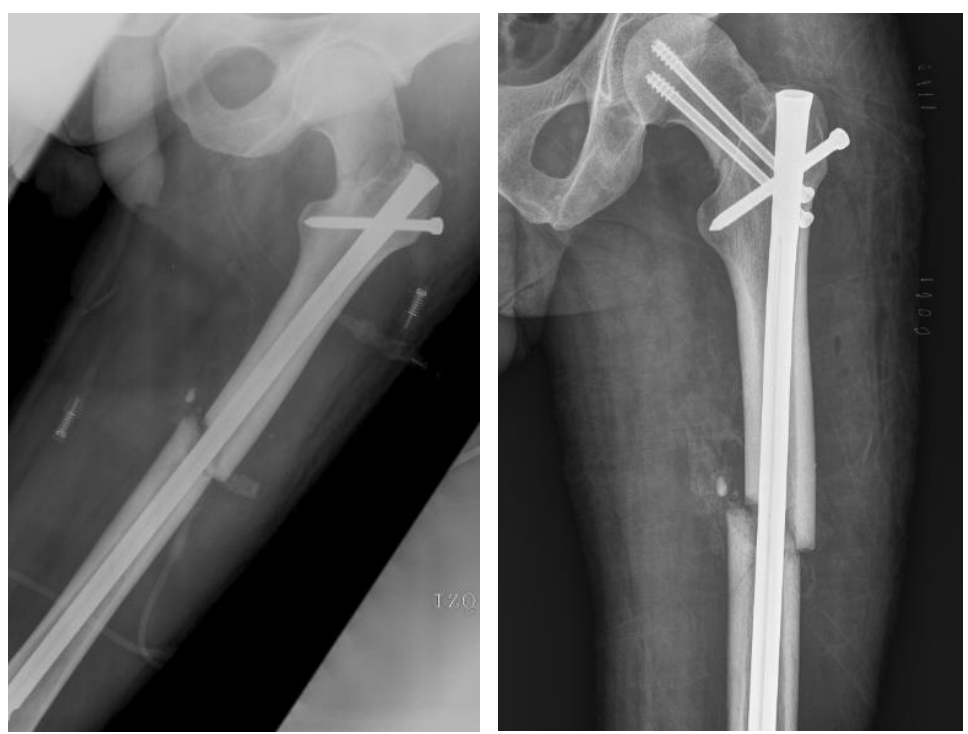

Figura 5: Clavo gross kempf. Se observa como hallazgo postoperatorio la aparición de fractura subcapital izquierda no diagnosticado en el momento previo a la fijación. En este caso, se optó por tornillos canulados en una segunda intervención.

En algunos casos, la reducción cerrada es fallida, por lo que es preciso utilizar la reducción abierta para estabilizar la cabeza previo a la fijación definitiva. Cuando se realiza la reducción abierta, entonces se utiliza la cabeza femoral como un joystic tras abrir la cápsula, y así se permite la correcta reducción [3]. En líneas generales, se tiende a utilizar la vía anterior directa (Smith Peterson) o bien la lateral (Hardinge), aunque esta es menos utilizada.

\section{Factores pronósticos y complicaciones}

Los factores asociados a buen pronóstico de la fijación son la cirugía precoz, la calidad de la reducción y la fijación estable. Los factores asociados a mal pronóstico son el desplazamiento y la conminución posterior [13]. 
Con respecto a las complicaciones, la ausencia de consolidación es más frecuente en la diáfisis femoral que en el cuello. El riesgo de ausencia de consolidación y osteonecrosis del cuello femoral depende de:

- Retraso en el diagnóstico

- Desplazamiento inicial

- Malreducción [13].

La incidencia de NAV y ausencia de consolidación es desconocida, pero se postula que aproximadamente el 5\% de las fracturas acabarán con necrosis avascular, y el 20\% con ausencia de consolidación, siendo este riesgo mayor si es una fractura abierta.

Se ha visto que la incidencia de NAV es menor que en el caso de fracturas de cuello femoral dado que se sospecha que la alta energía pueda disiparse por la diáfisis femoral, además de que muchas de las fracturas son no desplazadas, a diferencia de las fracturas aisladas de cuello femoral $[3,14]$.

Las tasas de mal unión se asociaban especialmente a un retraso de la fijación y a una mala reducción, así como conminución abundante. El posponer más de 48 horas antes de la cirugía no influía en la probabilidad de NAV cuando se comparaba con $>48$ horas tras cirugía. $[15,16,17]$

Sin embargo, la diáfisis tiene mayor tasa de complicaciones que si es aislada, dado que normalmente es más conminuta. El motivo por el cual la ausencia de consolidación pueda ser mayor que en las fracturas aisladas puede ser explicado porque son fracturas que han sufrido un mecanismo de alta energía, y además tienen una mayor tasa de fracturas abiertas comparado con las diafisarias aisladas. La afectación de partes blandas es la principal causa de esta complicación (Figura 6). [4,12]

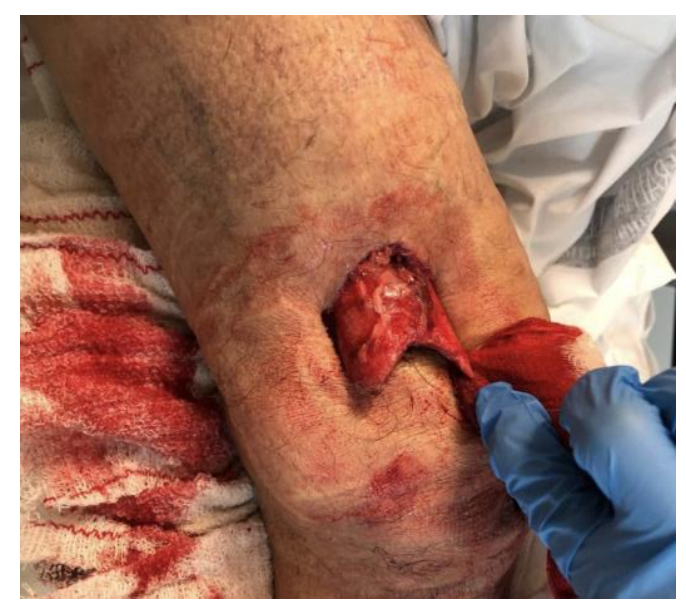

Figura 6: La afectación de partes blandas y las fracturas abiertas son el principal riesgo de ausencia de consolidación en la diáfisis. 
Se ha visto recientemente que las probabilidades de complicaciones en las fracturas ipsilaterales de cuello y diáfisis femorales son menores con clavo intramedular comparado con placa y tornillo [18].

Funding: Esta revisión no recibió financiación externa.

Conflicts of Interest: Los autores declaran que no existen conflictos de interés.

\section{References}

1. Alho A: Concurrent ipsilateral fractures of the hip and femoral shaft: A meta-analysis of 659 cases. Acta Orthop Scand 1996;67: 19-28.

2. Hak DJ, Mauffrey C, Hake M, Hammerberg M, Stahel PF: Ipsilateral femoral neck and shaft fractures: Current diagnostic and treatment strategies. Orthopedics 2015;38:247-251.

3. Jones CB, Walker JB. Diagnosis and Management of Ipsilateral Femoral Neck and Shaft Fractures. J Am Acad Orthop Surg. 2018 Nov 1;26(21):e448-e454

4. Singh R, Rohilla R, Magu NK, Siwach R, Kadian V, Sangwan SS. Ipsilateral femoral neck and shaft fractures: a retrospective analysis of two treatment methods. J Orthop Traumatol. 2008 Sep;9(3):141-7

5. Boulton CL, Pollak AN. Special topic: Ipsilateral femoral neck and shaft fractures--does evidence give us the answer? Injury. 2015 Mar;46(3):478-83

6. Yang KH, Han DY, Park HW, Kang HJ, Park JH. Fracture of the ipsilateral neck of the femur in shaft nailing. The role of CT in diagnosis. J Bone Joint Surg Br. 1998 Jul;80(4):673-8

7. Cannada LK, Viehe T, Cates CA, Norris RJ, Zura RD, Dedmond B, Obremskey W, Bosse MJ; Southeastern Fracture Consortium. A retrospective review of high-energy femoral neck-shaft fractures. J Orthop Trauma. 2009 Apr;23(4):25460.

8. Tornetta P 3rd, Kain MS, Creevy WR. Diagnosis of femoral neck fractures in patients with a femoral shaft fracture. Improvement with a standard protocol. J Bone Joint Surg Am. 2007 Jan;89(1):39-43.

9. Bedi A, Karunakar MA, Caron T, Sanders RW, Haidukewych GJ: Accuracy of reduction of ipsilateral femoral neck and shaft fractures: An analysis of various internal fixation strategies. J Orthop Trauma 2009;23:249-253.

10. Ostrum RF, Tornetta P 3rd, Watson JT, Christiano A, Vafek E. Ipsilateral proximal femur and shaft fractures treated with hip screws and a reamed retrograde intramedullary nail. Clin Orthop Relat Res. 2014 Sep;472(9):2751-8

11. Oh CW, Oh JK, Park BC, et al: Retrograde nailing with subsequent screw fixation for ipsilateral femoral shaft and neck fractures. Arch Orthop Trauma Surg 2006;126:448-453.

12. Cha YH, Yoo JI, Hwang SY, Kim KJ, Kim HY, Choy WS, Hwang SC. Biomechanical Evaluation of Internal Fixation of Pauwels Type III Femoral Neck Fractures: A Systematic Review of Various Fixation Methods. Clin Orthop Surg. 2019 Mar;11(1):1-14. doi: 10.4055/cios.2019.11.1.1. Epub 2019 Feb 18

13. Watson JT, Moed BR. Ipsilateral femoral neck and shaft fractures: complications and their treatment. Clin Orthop Relat Res. 2002 Jun;(399):78-86.

14. Upadhyay A, Jain P, Mishra P, Maini L, Gautum VK, Dhaon BK. Delayed internal fixation of fractures of the neck of the femur in young adults. A prospective, randomised study comparing closed and open reduction. J Bone Joint Surg Br. 2004 Sep;86(7):1035-40.

15. Araujo TP, Guimaraes TM, Andrade-Silva FB, Kojima KE, Silva Jdos S. Influence of time to surgery on the incidence of complications in femoral neck fracture treated with cannulated screws. Injury. 2014 Nov;45 Suppl 5:S36-9 
16. Wang T, Sun JY, Zha GC, Jiang T, You ZJ, Yuan DJ. Analysis of risk factors for femoral head necrosis after internal fixation in femoral neck fractures. Orthopedics. 2014 Dec;37(12):e1117-23. doi: 10.3928/01477447-20141124-60

17. Papakostidis C, Panagiotopoulos A, Piccioli A, Giannoudis PV. Timing of internal fixation of femoral neck fractures. A systematic review and meta-analysis of the final outcome. Injury. 2015 Mar;46(3):459-66.

18. Lu Y, Wang Y, Song Z, Wang Q, Sun L, Ren C, Xue H, Li Z, Zhang K, Hao D, Zhao Y, Ma T. Treatment comparison of femoral shaft with femoral neck fracture: a meta-analysis. J Orthop Surg Res. 2020 Jan 20;15(1):19. doi: 10.1186/s13018019-1496-z 